\title{
LA TEORÍA TEMÁTICA Y LAS MODALIDADES DE DESCARGA ARGUMENTAL: UNA PROPUESTA RESTRICTIVA
}

\author{
Guillermo Lorenzo González* \\ Universidad de Oviedo
}

\begin{abstract}
RESUMEN. El desarrollo de la Teoría Temática como uno de los módulo teóricos de la gramática generativa en su actual orientación ha venido a cooperar en la solución de uno de los problemas teóricos que primero fueron detectados dentro del antiguo modelo transformacional: los esquemas sintácticos básicos de la gramática debian ser cifrados dos veces, una en el componente de estructura de frase y otra en el marco de subcategorización de cada elemento léxico. El Modelo GB opta por la eliminación del primero de esos componentes y desarrolla la idea de que cada elemento léxico regula su desarrollo sintáctico de acuerdo con requisitos argumentales que se derivan de su contenido. En este trabajo se analiza cómo la Teoría Temática, sin embargo, ha experimentado en los últimos tiempos un proceso de desemantización que la ha permitido entrar en relación con ciertos fenómenos que en principio no parecian caer bajo su competencia.
\end{abstract}

ABSTRACT. The development of Thematic Theory as one of the modules of Generative Grammar has contributed to the solution of one the first problems detected in the old transformational model: the basic syntactic structures of the grammar were made explicit twice, in the phrase structure component and in the subcategorization frame of every lexical item. GB Model eliminates the first of these components and develops the idea that every lexical item controls its syntactic development in accordance with thematic requisites that derives from its meaning. This article analyzes, however, the progressive desemantization of Thematic Theory in the last times and how this permits its relation with facts before far from its competence.

1. Reglas y Principios. Desde sus primeras formulaciones, el generativismo fijó como objetivo básico de la teoría lingüística el desarrollo de mecanismos capaces de discriminar perfectamente las oraciones bien formadas de una lengua de aquellas otras

* Licenciado en Filología Española (Sección Lengua). Becario del Plan Nacional de Formación del Personal Investigador (Ref. PG8909393140). Area de Lingüística General, Departamento de Filología Española. Plaza Feijoo, 1 33003, Oviedo. 
que no pueden recibir tal consideración por parte de los hablantes (ver ya, en este sentido, CHOMSKY, 1957: 27). Sin embargo, la búsqueda de este objetivo no ha seguido un camino uniforme a lo largo de la historia del generativismo. Los primeros modelos generativistas (encarnados en CHOMSKY, 1957 y CHOMSKY, 1965) se caracterizaron por la formulación de la gramática de las lenguas, en primera instancia, como conjuntos de reglas, sometidas, en una segunda instancia, a una serie de transformaciones que, al tiempo que daban lugar a la manifestación superficial de los enunciados, permitían la detección de parentescos entre ellos en virtud de su vinculación a un conjunto común de reglas en el origen de su generación. Sin embargo, el modelo así planteado contenía el problema teórico de que a las reglas no cabía más que suponerles un origen arbitrario, por lo que, en principio, nada en la gramática misma servía para controlar ni su número ni su forma. Es decir, nada en la gramática permitía detener el paso de una gramática posible a una gramática imposible, de una gramática correspondiente a alguna lengua real a una gramática falta de esa correspondencia. En este sentido se dice hoy día que las gramáticas desarrolladas según aquellos modelos tenían excesivo poder generativo, o que su poder descriptivo excedía a las lenguas naturales que trataban de caracterizar.

La dirección que ha seguido la teoría lingüística a partir de los años ochenta para anular este problema ha sido la de concebir la gramática no como un conjunto de reglas, sino como un conjunto de principios encargados de limitar la forma de las gramáticas posibles. Esto no quiere decir exactamente que la gramática deje de poder formularse mediante reglas, sino que estas reglas no son elementos básicos de la gramática, sino objetos derivados de la aplicación de los principios (éstos sí, básicos) que regulan la gramaticalidad o agramaticalidad de los enunciados. Es decir, dado un conjunto reglas susceptibles todas ellas de ser interpretadas como objetos de descripción gramatical, la gramática propiamente dicha es el conjunto de principios capaz de decidir cuáles de esas reglas se aplican a las lenguas naturales y cuáles no (el primer modelo sistematizado en esta línea es CHOMSKY, 1981, que se conoce con el nombre de Gramática de Principios y Parámetros o Gramática GB).

Detengámonos en algunos ejemplos elementales que dan cuenta de la necesidad de este paso:

(1) ${ }^{*}$ María una vecina de mi hermano Miguel roció nuestro coche de gasolina.

(sin pausa entre "María" y "una vecina...")

$(2)^{*}$ Doné mis libros a mi gato a mi loro. (sin pausa entre "a mi gato" y "a mi loro")

Tanto (1) como (2) son, en efecto, oraciones agramaticales en español. En esta lengua podemos suponer, obviando ciertos detalles que no interesan aquí, la existencia de reglas como las de (3), pero no como las de (4), que son las que en realidad describirían la forma de las oraciones (1) y (2): 
(3) a. $\mathrm{O} \rightarrow \mathrm{SN}+\mathrm{SV}(\mathrm{V}+\mathrm{SN}+\mathrm{SP})$

b. $\mathrm{SV} \rightarrow \mathrm{V}+\mathrm{SN}+\mathrm{SP}$

(4) a. $\mathrm{O} \rightarrow \mathrm{SN}+\mathrm{SN}+\mathrm{SV}(\ldots)$

b. $\mathrm{SV} \rightarrow \mathrm{V}+\mathrm{SN}+\mathrm{SP}+\mathrm{SP}$

Sin embargo, nada en el tipo de gramática que presupone la formulación de (3) explica la imposibilidad de que en ella aparezcan reglas como las de (4), aparte de la pura formulación del conjunto de reglas. Es decir, sólo las reglas se explican a sí mismas, lo que en sí ya constituye una falta de explicación porque en ese caso estaríamos concediéndoles un carácter primitivo, estipulativo y, en definitiva, inexplicable. Además, puesto que los lenguajes naturales no son los únicos objetos susceptibles de ser interpretados mediante conjuntos de reglas, confiar a éstos todo el peso de la explicación lingüística no sirve para descubrir qué es lo propio del lenguaje humano frente a otros objetos afines. Es por esta razón por lo que el objeto de la lingüística debe ser antes la formulación de los principios que regulan la forma de las reglas que permiten describir las lenguas, que la exposición más o menos ordenada de las reglas mismas. De esta manera habremos confiado los primitivos de la explicación a objetos que pueden considerarse específicos del lenguaje humano y que no son compartidos por otros artefactos afines.

2. La Teoría Temática. Si retomamos ahora los ejemplos (1) y (2) y reflexionamos acerca del tipo de principio que incide en su agramaticalidad, no nos será difícil llegar a la conclusión de que su misión deberá ser la de evitar la duplicación (y, en general, la multiplicación) de Sintagmas Nominales desempeñando una misma función dentro de un único entorno oracional. Apurando algo más el contenido de esta observación y, sobre todo, ajustándola a otros principios de la gramática, nos interesará eliminar de ella la noción de "función", que tiene en el modelo generativo vigente un estatuto teórico secundario, derivado de otras nociones más básicas (véase, por ejemplo, SPEAS, 1990: 7-8). En efecto, el término "función" no nombra en la Gramática GB otra cosa que posiciones dentro de las jerarquías estructurales a las que da lugar el módulo categorial, más abstracto. De este modo, nociones como las de "sujeto", "complemento directo" o "complemento indirecto", por ejemplo, nombran respectivamente las posiciones en itálica dentro de los entornos $\left[_{\text {Esp }} S N\left[_{\text {Nucl }}\right.\right.$ Inf]], $\left.\left[_{\text {Nucl }} \mathrm{V}_{\text {Compl }} S N\right]\right]$ y $\left.\left[_{\text {Nucl }} \mathrm{V}_{\text {Compl }} S P\right]\right]$, legitimados por el módulo categorial de la gramática (o Teoría $\left.X^{\prime}\right)^{1}$.

$1 \mathrm{El} \mathrm{módulo} \mathrm{X'} \mathrm{de} \mathrm{la} \mathrm{gramática} \mathrm{es} \mathrm{el} \mathrm{heredero} \mathrm{de} \mathrm{las} \mathrm{reglas} \mathrm{de} \mathrm{estructura} \mathrm{de} \mathrm{frase} \mathrm{de} \mathrm{los} \mathrm{modelos} \mathrm{anteriores.} \mathrm{Ahora} \mathrm{bien,}$ con relación a aquellas guarda una diferencia radical, ya que todo ese componente pasa a articularse ahora en torno a dos únicos enunciados:

$$
\begin{aligned}
& \text { i. } X^{\prime \prime} \rightarrow \text { Espec(ificador) } X^{\prime} \\
& \text { ii. } X^{\prime} \rightarrow>\text { X Compl(ementos) }
\end{aligned}
$$

En estos dos enunciados aparecen codificadas varias ideas. En primer lugar, que todo sintagma es endocéntrico y que consiste en un núcleo susceptible de desarrollarse en dos niveles: un primer nivel en el que recibe complementos, y un segundo nivel en el que recibe un especificador. En segundo lugar, la existencia de regularidades entre elementos de categorias diferentes con relación a sus posibilidades de desarrollo sintagmático, lo que se expresa mediante la eliminación de toda etiqueta categorial en i. y ii. Cuáles sean los complementos o el tipo de los especificadores concretos que cada elemento recibe 
Lo que se busca, pues, es un principio que evite la multiplicación de esas posiciones. Una primera solución a la cuestión podría ser la de considerar que, puesto que tenemos un módulo que dicta las condiciones generales del desarrollo categorial, debería ser a él al que confiáramos también este tipo de limitaciones. Sin embargo, de este modo no habríamos conseguido dar un paso adelante en el tipo de gramática que se persigue. En efecto, si seguimos confiando al módulo categorial la especificación de los desarrollos sintagmáticos válidos para una lengua, por muy simple que sea este módulo respecto a las antiguas reglas de estructura de frase, no habremos dado ningún paso hacia una respuesta no autoevidente, es decir, no habremos enunciado ningún principio sobre el que hacer descansar la limitación que se persigue. Además, puesto que la reducción extrema del módulo categorial es posible gracias a la suposición de que la información que inicialmente codificaba se encuentra también, y necesariamente, codificada en el léxico, el diseño óptimo del módulo categorial será aquel en el que las posiciones que se encuentran a uno y otro lado del elemento nuclear en los diferentes niveles de proyección de una categoría no estén limiladas por el propio módulo. La razón es, naturalmente, que tales limitaciones estarán ya cifradas en la entrada léxica de cada unidad como condiciones de su desarrollo sintáctico.

Así pues, tenemos dos condiciones que deben cumplirse en el principio que se persigue, una de carácter negativo y la otra de tipo positivo:

i. se busca una solución no autoevidente, de mancra que no bastará con transcribir los entornos a los que dan lugar las unidades en términos de secuencias de entidades categoriales formando estructuras de frase; $y$

ii. la solución debe controlar más bien el desarrollo de tales secuencias en tanto que peculiaridades léxicas asociadas a las unidades que las promueven.

2.1. La formulación inicial de la Teoría- $\theta$. Creemos que este minucioso razonamiento debe servir para entender el tipo de solución, el principio, que se propuso para solventar este problema: la llamada Teoría Temática (o Teoría- $\theta$ ). La Teoría- $\theta$ cuenta ya con una larga historia que la ha hecho atravesar una serie de estadios no coincidentes entre sí, en cada uno de los cuales la Teoría se ha depurado o se ha desarrollado hasta hacerla extensible a fenómenos con los que, en principio, no guardaba relación. En su formulación inicial, la Teoría- $\theta$ se concebía como un módulo de la gramática de carácter semántico, aunque atravesado por un regulador de tipe formal, que no era otro que la propia Teoría X' (CHOMSKY, 1981). A partir de aquí la Teoría ha ido evolucionando básicamente en dos direcciones: en la relativización de su carácter semántico (como en JACKENDOFF, 1987, RAPPAPORT-LEVIN, 1988 o GRIMSHAW, 1990) y en su extensión a fenómenos que inicialmente no cubría (como en HIGGINBOTHAM, 1985

\footnotetext{
es algo que debe codificarse independientemente en la entrada léxica de cada unidad, por loque su incorporación en el módulo de estructura de frase o módulo categorial seria completamente redundante respecto a aquellas (véase CHOMSKY, 1981:3132 y STOWELL, 1981 : 9-51; una propuesta de total eliminación del módulo categorial como primitivo de la gramática se encuentra en FUKUI-SPEAS, 1986, y se justifica estructural y semánticamente en LORENZO, 1991b).
} 
o SPEAS, 1990).

La Teoría- $\theta$ tiene su fundamento último en la Hipótesis Léxica que subyace a todo el modelo gramatical GB. Esta hipótesis, según la cual en el léxico se encuentra el motor de todo el proceso generativo, se origina en el momento en el que se apreció que prácticamente toda la información que se venía codificando en el nivel de representación de Estructura de Frase resultaba redundante con información que, en cualquier caso, era necesario vincular a peculiaridades léxicas de las unidades ${ }^{2}$. De este modo, las peculiaridades de los entornos sintácticos en los que aparece un determinado elemento pasó a concebirse como dependiente de sus peculiaridades léxicas (o de los elementos vecinos), y no como originada en un componente en el que las piezas léxicas se integraban únicamente como elementos terminales.

Además, como señalan Chomsky, no sin ambigüedad, y más abiertamente Williams y Lasnik y Uriagereka (CHOMSKY, 1981: 42; LASNIK-URIAGEREKA, 1988: 4; WILLIAMS, 1981: 89), el factor que incide fundamentalmente en las peculiaridades de subcategorización de una pieza son sus características léxicas inherentes (lo que acostumbramos a llamar su contenido semántico o su significado). Por consiguiente, la subcategorización de esquemas sintácticos parece derivarse de la selección, en primer término, de ciertos tipos de "ramificaciones semánticas" por parte de las piezas léxicas. Para estas "ramificaciones" la teoría gramatical de los años 60 y 70 ya contaba con una noción, la de papel temático ("agente", "tema", "beneficiario",...), a la que se venía concediendo un papel interpretativo, pero que se elevará ahora a la categoría de base generativa, es decir, de objeto de la Estructura Profunda ${ }^{3}$.

En suma, el nuevo rumbo que adopta la teoría gramatical determina que las representaciones en los diferentes niveles se proyectan desde el léxico, ya que deben observar las peculiaridades de subcategorización de las piezas, y que cada una de las posiciones que satisfacen estas peculiaridades es una posición temática (o posición- $\theta$ ). Y, en definitiva, que la Estructura Profunda es, de entrada, una representación de la asignación de papeles- $\theta$ por parte de las piezas léxicas (CHOMSKY, 1981: 29-36).

Por tanto, dados estos presupuestos básicos de la tcoría, la agramaticalidad de los ejemplos (1) y (2) debe estar regulada por una disposición que, a las condiciones expuestas en i. y ii., sume la de referirse a los objetos conceptuales de tales presupuestos. En efecto, el Criterio- $\theta$ de Chomsky cumple esas condiciones una a una. Dice así:

2. En efecto, aunque el componente de Estructura de Frase contara con una regla como "SV -> V SN SP", en la entrada léxica de una unidad concreta seguía siendo necesario especificar que el entomo sintáctico al que daba lugar era éste y no, pongamos por caso, "SV $\rightarrow$ V SN", también contenido en aquel componente. De este modo, evidentemente, ese tipo de informaciones aparecía transcrita en dos componentes diferentes de la gramática: en el de Estructura de Frase y en el Léxico (véase un desarrollo de esta critica en HENY, 1979 y una acogida, implícita, de la misma en CHOMSKY, 1981 : 21-32). Como, en cualquier caso, los lexemas deben aclarar los entornos que originan, la vía que quedaba para eliminar esta redundancia de la gramática era la de revisar el componente de Estructura de Frase (aunque, como es sabido, la GPSG optó por el camino contrario: mantener este componente y elaborar un mecanismo de coindización capaz de vincular cada pieza léxica con uno de los esquemas sintácticos previstos en él; véase GAZDAR-KLEIN-PULLUM-SAG, 1985: 31-35).

3. Ese valor interpretativo es el que se le concede, por ejemplo, en JACKENDOFF, 1972. Su papel en la Gramática GB se parece más al que se desarrollaba en modelos como los de FILLMORE, 1968 y GRUBER, 1976. 
(5) Todo argumento tiene un solo papel- $\theta$ y todo papel- $\theta$ se asigna a un solo argumento (CHOMSKY, 1981: 36).

El Criterio- $\theta$ representa la condición básica de buena formación semántica de los enunciados. A esta condición de tipo semántico, la Teoría- $\theta$ debe añadir una condición formal de buena formación, que, como ya dijimos, le viene dada por el módulo categorial o Teoría X' (CHOMSKY, 1981: 35-36). Esta condición formal determina, básicamente, que los diferentes argumentos de una unidad deben establecerse como complementos suyos, a excepción de uno de ellos (llamado externo por Williams) que aparece marcado al efecto de poder situarse fuera de este ámbito y elevarse hasta una proyección superior y externa a la unidad que lo sclecciona como especificador ${ }^{4}$ (WILLIAMS, 1981: 83-84).

2.2. La relativización del carácter semántico de la Teoría- $\theta$. Una entrada léxica que respetase los postulados de la Teoría- $\theta$ vistos hasta aquí tendría una forma como la siguiente:

(6) Donar < AGENTE, TEMA, BENEFICIARIO>

(donde el subrayado, siguiendo la convención de Williams, señala el argumento externo $\mathrm{y}_{\mathrm{a}_{\mathrm{a}} \text { " }}$ transcribe la regla de realización del argumento BENEFICIARIO que determina la unidad "donar")

En este tipo de entrada los papeles- $\theta$ cumplen dos objetivos diferentes: en primer término, determinan el número de argumentos que deben aparecer satisfechos en el enunciado para que pueda considerarse correcto desde el punto de vista gramatical y, en segundo término, explicitan el contenido semántico de la relación que cada uno de esos argumentos guarda con respecto al elemento que los selecciona. Es en este sentido en el que puede decirse que la Teoría- $\theta$, tal como se concibe en CHOMSKY, 1981, tiene un carácter híbrido, entre semántico y sintáctico.

Sin embargo, aunque resulta evidente que los dos aspectos recogidos en la noción de papel- $\theta$ supuesta en ese tipo de entradas léxicas son pertinentes a efectos lingüísticos, no lo es tanto que ambos lo sean en el mismo nivel y con el mismo carácter. En efecto, parece claro que la etiqueta semántica concre ta que cada argumento recibe en un entorno de selección como (6) no tiene ninguna incidencia en la dimensión puramente sintáctica de la Teoría- $\theta$, es decir, no influye, por ejemplo, en una mayor o menor rigidez en la condición de saturación argumental de los papeles temáticos seleccionados (ni tampo-

4. Salvo, claro está, que medien ciertos procesos morfológicos que alteren la jerarquía inicial. Por lo demás, a cada tipo de argumento se le puede suponer una categoría gramatical canónica, lo que evita su especificación en cada entrada léxica concreta. No obstante, parecenecesario contar con un mecanismo que de cuenta de los detalles de la manifestación superficial de los argumentos, así como de las alteraciones que puedan presentar deteminadas piezas léxicas respecto a las opciones canónicas o por defecto. Este es el sentido de las reglas de realización en WILLIAMS, 1981 o los mecanismos previstos en la Estructura Léxico-Sintáctica de ZUBIZARRETA, 1987. 
co, naturalmente, en la posibilidad de multiplicar el número de sintagmas que los saturan). En definitiva, el contenido asociado a los papeles temáticos es completamente independiente de las condiciones sintácticas que imponen a los enunciados 5 .

Este es, pues, un primer argumento para rechazar que las etiquetas semánticas de los papeles- $\theta$ sean pertinentes con relación al problema que suscitó el desarrollo de la Teoría Temática: puesto que su contenido específico no parece ejercer ninguna influencia sobre las condiciones que la teoría impone a los enunciados, parece que en su formulación puede bastar una pura especificación numérica de los argumentos que deben manifestarse en el enunciado. Esto explica la forma que pasa a adoptar la dimensión de una entrada léxica pertinente a efectos de la Tcoría- $\theta$, en la que las etiquetas de los papeles- $\theta$ dan paso a simples variables, como en ZUBIZARRETA, 1987, o números, como en HIGGINBOTHAM, 1985:

(7) Donar-x, y; (Ben a-z) (donde "-" indica que el argumento que aparece a su derecha es complemento de la unidad que aparece a su izquierda, y los paréntesis la opcionalidad de ese argumento; "Ben" especifica que, en este caso, la preposición "a" tiene el valor "beneficiario", excluyéndose cualquier otro de aquellos a los que también puede aparecer asociado)

(8) Donar $<1,2,3>$

Las entradas se despojan, de este modo, de su componente semántico y se convierten en puros objetos sintácticos. Naturalmente, la descripción del contenido inherente de las unidades sigue siendo pertinente en otro nivel de representación, pero se descarta que éste tenga incidencia directa sobre un regulador sintáctico como es el Criterio- $\theta$.

¿Qué valor tiene entonces, si es que tiene alguno, o de dónde procede el contenido que tradicionalmente se venía asociando a los papeles- $\theta$, reducidos ahora a puros espacios argumentales desemantizados? Debemos tener en cuenta que un segundo argumento encaminado a restar pertinencia sintáctica a los papeles- $\theta$ ha consistido en demostrar que tales etiquetas no son, de nuevo, elementos básicos de la teoría lingüística, sino objetos derivables de otros componentes teóricos aún más básicos ${ }^{6}$.

Efectivamente, todo lo que hemos venido exponiendo no quicre decir que el significado de las unidades carezca de valor linguístico, sino únicamente que esta dimensión no debe confundirse con la dimensión sintáctica, de modo que una y otra deben recogerse en módulos de representación diferentes. Por tanto, si una entrada léxica

5. Tampoco inciden, como señalan Rappaport y Levin, en las reglas de realización de los argumentos (RAPPAPORTLEVIN, 1988: 13-16).

6. Se puede encontrar una síntesis del debate acerca del carácter primitivo o derivado de los papeles- $\theta$ en CULLICOVER, 1987. 
debe constar, como se ha visto, de un componente sintáctico, tampoco ha de faltarle una especificación de su contenido semántico inherente, aunque es una condición que actúa sobre la forma de las entradas léxicas el que no se confundan en la representación uno y otro aspecto. No obstante, aunque no se confundan, lo que sí parece claro es que deben relacionarse de algún modo, porque, en definitiva, debe ser el contenido inherente de una unidad el que actúe como fuente semántica de los argumentos.

La propuesta más clara para relacionar uno y otro nivel, satisfaciendo a un tiempo la condición de no identificación, es la que puede encontrar en RAPPAPORT-LEVIN, 1988. Rappaport y Levin proponen un primer nivel de representación al que denominan Estructura Léxico Conceptual (Lexical Conceptual Structure, LCS), consistente en una suerte de definición en la que una parte del contenido de la unidad se expresa mediante variables que indican las relaciones que ese contenido implica. (9) es un ejemplo de LCS:

(9) LCS para CORTAR: $x$ provoca una separación lineal sobre la integridad material de $y$ mediante la aplicación de un filo sobre aquella.

Un segundo nivel de representación, que Rappaport y Levin denominan Estructura de Predicado-Argumentos (Predicate Argument Structure, PAS), recoge únicamente los aspectos relacionales de la LCS pertinentes en la sintaxis ${ }^{7}$. La PAS correspondiente a la LCS (9) es la siguiente:

\section{(10) PAS para CORTAR: $<1,2>$}

Considerando estas dos dimensiones en una entrada léxica, salta inmediatamente a la vista el carácter derivado de las etiquetas que nombran a los diferentes papeles- $\theta$ : no nombran, en realidad, más que la asociación de una determinada variable argumental en la PAS con una variable en la LCS, y se derivan, por tanto, de ciertas características generales de los contenidos expresados en ésta ${ }^{8}$.

2.3. La expansión de la Teoría- $\theta$. Puede decirse, en suma, que la Teoría- $\theta$ ha experimentado un proceso de desemantización que conduce a su situación actual, en la

7. Para hacer realmente operativa una propuesta como la de Rappaport y Levin es necesario el cumplimiento de ciertas condiciones básicas: en primer lugar, las LCSs deben ser susceptibles de articularse en un lenguaje consistente en un número lo más reducido posible de elementos conceptuales básicos, cada uno de ellos con ciertas características de tipo relacional; y, en segundo lugar, de su encadenamiento deben poder derivarse de manera automática las características relacionales (PAS) de las unidades que definen las LCSs. En este sentido, naturalmente, (9) es una LCS todavia demasiado burda. Sobre estas condiciones y ciertos caminos que cabe seguir en su cumplimiento véase LORENZO, 1991a.

8. Una propuesta semejante, en sus asunciones básicas, a la de Rappaport y Levin es la de JACKENDOFF, 1987, donde se estipula un nivel de representación semántico-conceptual y un nivel de estructuras argumentales que no hace sino abreviar los aspectos del primero "visibles" a la sintaxis. También en este modelo se desprende sin dificultad que las etiquetas de los papeles- $\theta$ no son más que convenciones mnemotécnicas para ciertas configuraciones estructurales de las representaciones semántico-conceptuales. 
que la teoría maneja lo que se denominan estructuras argumentales. Una estructura argumental, en el sentido establecido en GRIMSHAW, 1990, consiste en una pura especificación numérica de las características de subcategorización de una pieza léxica, dotada, eso sí, de cierta organización interna: esta organización consiste básicamente en la disposición jerárquica de sus miembros, que trata de dar cuenta de la diferente propensión que tiene cada uno de ellos a ocupar posiciones estructurales privilegiadas (GRIMSHAW, 1990: 33-37).

Pero la Teoría- $\theta$ ha experimentado un segundo proceso de revisión en virtud del cual se ha hecho depender de ella fenómenos que en principio le eran ajenos. Inicialmente, la Teoría- $\theta$ se encargó de regular únicamente la saturación y la no multiplicación de los argumentos de las piezas léxicas, derivados de sus características relacionales. Sin embargo, a partir de HIGGINBOTHAM, 1985, pasó a considerarse que también debían ser competencia de la misma teoría un conjunto de hasta tres fenómenos gramaticales, además del que inicialmente cubría, que pasa a denominarse marcado- $\theta$ ( $\theta$-marking).

En primer lugar, Higginbotham observa que los nombres, en muchas lenguas, presentan las siguientes propiedades: en primer lugar, la de poder servir como predicados y, en segundo lugar, la de tener que saturar, en caso contrario, la posición de especificador de su proyección máxima. Una manera de relacionar estas dos propiedades, conviertiéndolas de hecho en una sola, consiste en suponer que en la estructura argumental de estas unidades existe un argumento suplementario, e igualmente pertinente a efectos del Criterio- $\theta$, que debe saturarse ya sea por vía de su coindización con un sujeto (y el nombre actúa, entonces, como predicado), ya sea mediante la saturación del especificador de $\mathrm{N}$ ". A esta modalidad de descarga argumental la denomina Higginbotham ligamiento- $\theta$ ( $\theta$-binding) (HIGGINBOTHAM, 1985: 560).

En segundo lugar, Higginbotham señala que una buena manera de explicar el efecto semántico de adición de propiedades que se detecta en buena parte de los casos de modificación de los nombres a cargo de los adjetivos ${ }^{9}$, es la de suponer la existencia de posiciones en la estructura argumental de una y otra categoría talcs que se saturan, cada una de ellas, en el encuentro con la otra. A esta modalidad de descarga argumental, asociada al efecto semántico mencionado, es a la que Higginbotham denomina identificación- $\theta$ ( $\theta$-indentification) (HIGGINBOTHAM, 1985: 565-564).

Finalmente, la introducción de la última modalidad de descarga argumental mencionada parece imponer la consideración de una modalidad más: la que se registra en configuraciones estructurales idénticas a la de la modalidad anterior, pero sin que de la descarga se siga el efecto de adición de las propiedades ${ }^{10}$. Higginbotham llama a esta última modalidad marcado- $\theta$ autónimo (autonymous $\theta$-marking) (HIGGINBOTHAM, 1985: 564-565).

9. Es el efecto semántico que se registra, por ejemplo, en casos como "casa blanca": de una entidad a la que se aplique se podrá decir que es una casa y que es blanca. Lógicamente se suele representar este cfecto como la conjunción de las dos propiedades: "casa $(\mathrm{x})$ \& blanca $(\mathrm{x})$ ".

10. Como, por ejemplo, en "presunto delincuente", del que no cabe decir que su representación lógica sea "presunto (x) \& delincuente $(x)^{\prime \prime}$. 
Con Higginbotham, en definitiva, la Teoría Temática crece hasta cubrir un total de cuatro fenómenos, que se unifican ahora por el rasgo común de depender de alguna de las modalidades de descarga argumental. Por tanto, el Criterio- $\theta$ no debe ocuparse únicamente, en opinión de Higginbotham, de las condiciones que regulan la saturación de las variables argumentales en una estructura temática (marcado- $\theta$ ), sino también de la de otra serie de variables que no deben considerarse exactamente argumentales. Por esta razón, Higginbotham prefiere hablar de posiciones temáticas (donde "temáticas" carece de implicaciones semánticas) para unificar terminológicamente al conjunto de variables que deben saturarse en una red temática (término que prefiere al de "estructura argumental"). En consecuencia, Higginbotham revisa el Criterio- $\theta$, que pasa articularse en dos enunciados con los siguientes términos:

(11)

i. Toda posición temática se descarga, y

ii. si $X$ descarga un papel temático en $Y$, entonces descarga sólo uno. (HIGGINBOTHAM, 1985: 561)

\section{Una propuesta restrictiva acerca de las modalidades de descarga argumen-} tal. Una primera observación que cabe hacer a propósito de las diferentes modalidades de descarga argumental previstas por Higginbotham, es de que, con ellas, la Teoría- $\theta$ vuelve a semantizarse: efectivamente, las modalidades de identificación- $\theta$ y marcado$\theta$ autónimo implican que un efecto de carácter semántico corre paralelo a la descarga argumental propiamente dicha. En este sentido, el trabajo de Higginbotham recibe una crítica en SPEAS, 1990, tendente a liberar nuevamente el fenómeno de descarga argumental de todo resquicio semántico.

Speas, ateniéndose al modelo teórico diseñado por Rappaport y Levin, defiende que los efectos semánticos que Higginbotham vincula a los fenómenos de descarga son susceptibles de desplazarse, de nuevo, hasta el nivel de LCS. Para Speas, que la identificación- $\theta$ dé lugar a la adición de propiedades y que el marcado- $\theta$ autónimo no lo haga y se acompañe de efectos semánticos de otro género (semejantes a los que la semántica modelo-teórica ilustra como casos de contextos intensionales), se debe al contenido de las LCSs de las unidades que dan lugar a una u otra modalidad de descarga (SPEAS, 1990: 70). Por ejemplo, Speas argumenta que a una pieza léxica como "blanco" cabe suponerle una LCS como (12), que autoriza la adición, al no disponer nada en sentido contrario, y una PAS como (13), con una posición temática que se descarga sobre un nombre:

(12) LCS para BLANCO: $x$ carcce de color y es opaco

(13) PAS para BLANCO: $<1>$

En cambio, la LCS de "presunto" sí pone freno a la adición, al tiempo que su PAS 
no difiere de la de "blanco":

(14) LCS para PRESUNTO: se considera que $\mathrm{P}(\mathrm{x})$

(donde " $P$ " nombra una propiedad, con relación a la cual se produce la descarga, que en segunda instancia se aplica a una entidad)

\section{(15) PAS para PRESUNTO: $<1>$}

En consecuencia, la descarga argumental propiamente dicha no difiere en nada en uno u otro tipo de unidades, y el efecto semántico que Higginbotham les asocia procede en realidad del entorno en el que se encuentra en la LCS la variable que experimenta la descarga.

Tras esta revisión, el universo de modalidades de descarga queda reducido a tres miembros: marcado- $\theta$, ligamiento- $\theta$ e identificación- $\theta$, manteniendo la terminología de Higginbotham ${ }^{11}$. Sin embargo, nuestra opinión es que cabe ir más allá y defender que los rasgos que diferencian a estas modalidades entre si no proceden de las modalidades en sí, sino de los entornos en los que se aplican y el tipo de unidades que aparecen en ellos. De ser así, no habrá más que una sola modalidad de descarga argumental y, una vez más, las posibles variantes que estemos dispuestos a considerar no serán objetos básicos de la teoría, sino derivados de la confluencia de otras nociones más básicas.

3.1. Contra el ligamiento- $\theta$. En contra de la modalidad de ligamiento- $\theta$ vamos a manejar nuestra creencia en la existencia de lo que se viene llamando Sintagmas Determinantes. La categoría de Sintagma Determinante, puesta en circulación por Brame (BRAME, 1982) y más ampliamente razonada por Abney como proyección de Det, una de las Categorías Funcionales junto a Compe Infl (ABNEY, 1986), encuentra justificaciones tanto desde el punto de vista estructural como semántico ${ }^{12}$. El argumento sobre el que descansa la introducción de la categoría por parte de Brame, que asume y explicita Abney (BRAME, 1982: 321-322; ABNEY, 1986: 8), es el de la direccionalidad de la subcategorización y, en concreto, que en lenguas como el inglés o el español tiene lugar hacia la derecha. Desde este presupuesto, debe ser Det quien subcategorice a $\mathrm{N}, \mathrm{y}$ no a la inversa. Otro argumento importante es el que se deriva de ejemplos como "el viejo" o"cl alto", para los que se necesitan reglas de estructura de frase del tipo "SN -> Det A", que plantean un importante problema teórico: el elemento que aparece a la izquierda no es proyección de ninguna de las categorías que aparecen a la derecha. Si suponemos, en cambio, que la regla es más bien "SDet $\rightarrow$ Det P", donde "P" significa "propiedad", es decir, $\mathrm{N}$ o A, el problema se soluciona sin atenernos a procedimientos de transposición, ya que el elemento de la izquierda es proyección del elemento nuclear

11. Speas revisa además la terminología y habla de descarga (discharge $=$ marcado- $\theta$ ), emergencia (merger $=$ identificación- $\theta$ ) y ligamiento (binding = ligamiento- $\theta$ ) (SPEAS, 1991:71).

12. En LORENZO, 1991 b, se encuentra una pormenorizada relación de argumentos tanto acerca de la especificidad categorial de Det, como de su carácter nuclear en proyecciones sintagmáticas en las que los nombres tienen un carácter subordinado y ocupan la posición de complemento. 
Det, que sí aparece a la derecha.

Pero creemos que el argumento más importante es de otro género. Sabemos que la actualización es una condición necesaria que se debe cumplir para que un SN pueda aparecer como argumento en una oración y que esta condición sólo se satisface mediante la mediación de los miembros de la categoría Det. Así pues, puede concluirse que la subcategorización de argumentos se relaciona antes con la categoría Det que con la categoría N: es decir, los complejos sintagmáticos que saturan los espacios argumentales de una fórmula deben ser proyecciones de la categoría Det ${ }^{13}$.

A toda esta serie de pruebas ${ }^{14}$, cabe sumar los síntomas que, para Abney, sugieren que Det es efectivamente una categoría semejante a Comp o Infl, es decir, una Categoría Funcional:

i. su inventario es cerrado;

ii. se puede decir que sus miembros carecen, hablando propiamente, de contenido inherente; $y$

iii. su función semántica es afín: sirven para fijar la referencia de algún otro tipo de categoría semántica, que en el caso de Det son los nombres y los adjetivos, es decir, las propiedades. (ABNEY, 1986: 8-9)

En definitiva, parece que existen razones, muy numerosas e independientes al problema que tratamos aquí, que permiten defender la existencia de Sintagmas Determinantes.

Puesto que en HIGGINBOTHAM, 1985, se sigue asumiendo que los determinantes son los especificadores de las proyecciones $\mathrm{N}$ " ( $\mathrm{y}$, por tanto, que la noción de "determinante" nombra únicamente una configuración estructural y no una categoría), se suponen dependientes, en cuanto a su generación, de los nombres, núcleos a partir de los cuales se proyecta el nivel de desarrollo categorial del que forman parte. Es lógico, por tanto, que el ligamiento- $\theta$ se considere una modalidad de descarga que parte del nombre, nuclear, y alcanza al determinante, subordinado.

En cambio, según los presupuestos que nosotros defendemos son los determinantes los que subcategorizan a los nombres, es decir, los que tienen carácter nuclear con relación a los últimos. Por tanto, la descarga debe tener más bien el sentido contrario: partir de los determinantes y alcanzar a los sustantivos, consumándose entonces su saturación. De este modo es fácil proceder a una nueva simplificación de las modalida-

13. Esta opinión está en la línea, defendida por Baltin, de que la subcategorización tiene lugar, en primer término, sobre los núcleos, y no sobre las proyecciones máximas (BALTIN, 1989:4). Sin embargo, Baltin niega curiosamente, en ese mismo trabajo, la existencia de Sintagmas Deteminantes.

14. A las que cabría añadir las de Stowell, que opina que la introducciön de los Sintagmas Determinantes en el panorama categorial tiene el efecto de explicar ciertos hechos estructurales relativos a la distribución de PRO y el movimiento de piezas a COMP (STOWELL, 1989). 
des de descarga argumental: puesto que el marcado- $\theta$ y el ligamiento- $\theta$ se distribuyen complementariamente según tipos de categorías (el primero afecta a las Categorías Léxicas y el segundo a las Funcionales ${ }^{15}$ ), podemos considerar que se trata de una modalidad única, ya que las peculiaridad que cada una muestra con relación a la otra no procede de nada intrínseco a la modalidad en sí, sino del tipo de categoría con la que se vincula. Esta peculiaridad es, en esencia, que la descarga se vincula en las Categorías Léxicas con variables susceptibles de ocupar en la LCS una de las posiciones que se sintetizan mediante un papel- $\theta$, mientras que en las Categorías Funcionales no existen ese tipo de posiciones ${ }^{16}$.

3.2. En contra de la identificación- $\theta$ y conclusión. No es difícil claborar un argumento semejante al anterior encaminado a anular la especificidad de la modalidad de identificación- $\theta$. Esta modalidad de descarga es la que se vincula a los adjetivos, es decir, a las propiedades de segundo orden que saturan su dimensión relacional en la dependencia respecto a propiedades de primer orden, es decir, respecto a nombres, en el seno de las proyecciones de éstos. Por tanto, cabe argumentar, una vez más, que lo específico no es la modalidad de descarga, sino el entorno categorial en el que tiene lugar.

En conclusión, cabe mantener que existe una única modalidad de descarga argumental, por lo que es mejor no hablar de "modalidad", sino de "fenómeno" de descarga. Este fenómeno único puede, eso sí, adquirir tintes relacionales y semánticos diversos en función de los diferentes entornos en los que puede desarrollarse. En cambio, lo que parece necesario aumentar es el número concreto de espacios argumentales que deben figurar en una estructura argumental, puesto que el Criterio- $\theta$ no se aplica únicamente a aquellos que tradicionalmente venían asociados a los papeles- $\theta$. No obstante, puesto que una estructura argumental debe considerarse como una pura especificación numérica de las propiedades de subcategorización de una pieza léxica, su crecimiento no va aparejado a una multiplicación de las entidades que debe manejar la teoría.

\section{REFERENCIAS}

ABNEY, S. (1986).- "Functional elements and licensing". Comunicación presentada en la GLOW Conference, Barcelona.

BALTIN, M.R. (1989).- "Heads and projections". Alternative Conceptions of Phrase Structure (ed. by M.R. Baltin \& A.S. Kroch). The University of Chicago Press

15. Asumimos que este tipo de subcategorización es el que liga también a Comp e Infl con sus complementos, I' y $V^{\prime \prime}$, respectivamente (véase ABNEY, 1986: 8).

16. Siguiendo literalmente a Abney cabría decir que el ligamiento- $\theta$ no es más que una noción derivada para nombrar la modalidad de descarga argumental asociada a categorias sin LCS, ya que defiende que las Categorias Funcionales carecen de contenido inherente. Sin embargo, creemos que sí es necesario vincular a los miembros de Det, Comp e Infl, con ciertos contenidos inherentes susceptibles de codificarse en LCSs. 
(pp. 1-16).

BRAME, M. (1982).- "The Head-Selector Theory of Lexical Specifications and the nonexistence of coarse categories". Linguistic Analysis, v.10/n.4 (pp. 321-325).

CHOMSKY, A.N. (1957).- Syntactic Structures. The Hague, Mouton (versión española de C.P. Otero: Estructuras Sintácticas. México, Siglo XXI, 1987).

CHOMSKY, A.N. (1965).-Aspects of the Theory of Syntax. Cambridge(MA), The MIT Press.

CHOMSKY, A.N. (1981).- Lectures on Government and Binding. Dordrecht, Foris Publications.

CULLICOVER, P. (1987).- "On thematic relations". MIT Working Papers in Linguistics, v.9 (pp. 65-92).

FILLMORE, CH.J. (1968).- "The case for case". Universals in Linguistic Theory (ed, by E. Bach and R. Harms). New York, Holt, Rcinhart \& Winston (pp. 1-88).

FUKUI, N. \& SPEAS, M. (1986).- "Specifiers and Projection". MIT Working Papers in Linguistics, v.8 (pp. 128-172).

GAZDAR, G.; KLEIN, E.; PULLUM, G. \& SAG, I. (1985).- Generalized Phrase Structure Grammar. Cambridge (MA), Harvard University Press.

GRIMSHAW, J. (1990).- Argument Structure. Cambridge (MA), The MIT Press.

GRUBER, J.S. (1976).- Lexical Structures in Syntax and Semantics. Amsterdam, North-Holland.

HENY, F. (1979).- "Review Article (on Chomsky, Logical Structure of Linguistic Theory)". Synthese, 40 (pp. 317-352).

HIGGINBOTHAM, J. (1985). - "On Semantics". Linguistic Inquiry, v.16-n.4 (pp. $547-$ 593).

JACKENDOFF, R. (1972).- Semantic Interpretation in Generative Grammar. Cambridge (MA), The MIT Press.

JACKENDOFF, R. (1987).- "The status of thematic rclations in linguistic theory". Linguistic Inquiry, v.18 / n.3 (pp. 369-411).

LASNIK, H. \& URIAGEREKA, J. (1988).- A Course in GB Syntax. Cambridge (MA), The MIT Press.

LORENZO, G. (1991a).- "La hipótesis léxica en la Gramática GB: problemas y alternativas". Sintagma, 3 (pp. 41-51).

LORENZO, G. (1991b).- C-3PO: Una teoría general de la referencia con aplicación a los Sintagmas Determinantes. Memoria de Investigación inédita, Universidad de Oviedo.

RAPPAPORT, M. \& LEVIN, B. (1988).- "What to do with $\theta$-roles?". Syntax and Semantics, 21 : Thematic Relations (ed. by W. Wilkings). San Diego, Academic Press (pp.7-36).

SPEAS, M. (1990).- Phrase Structure in Natural Language. Dordrecht, Kluwer Academic Publishers.

STOWELL, T. (1981).- Origins of Phrase Structure. Unpublished Ph.D. Dissertation, MIT.

STOWELL, T. (1989).- "Subjects, specifiers, and X-Bar Theory". Alternative Concep- 
TEORÍA TEMÁTICA Y MODALIDADES DE DESCARGA ARGUMENTAL: UNA PROPUESTA RESTRICTIVA

tions of Phrase Structure (ed. by M.R. Baltin \& A.S. Kroch). The University of Chicago Press (pp. 232-262).

WILLIAMS, E. (1981).- "Argument structure and morphology". The Linguistic Review, 1.1 (pp. 81-144).

ZUBIZARRETA, M.L. (1987).- Levels of Representation in the Lexicon and in the Syntax. Dordrecht, Foris Publications. 